\title{
XIX. A study of the ionization produced by $\beta$ and $\gamma$ rays at high pressures
}

\section{D.C.H. Florance M.A. M.Sc.}

To cite this article: D.C.H. Florance M.A. M.Sc. (1913) XIX. A study of the ionization produced by $\beta$ and $\gamma$ rays at high pressures, Philosophical Magazine Series 6, 25:145, 172-183, DOI: 10.1080/14786440108634322

To link to this article: http://dx.doi.org/10.1080/14786440108634322

$$
\text { 曲 Published online: } 20 \text { Apr } 2009 .
$$

\section{Submit your article to this journal $\sqsubset$}

Џ Article views: 4

Q View related articles $\sqsubset$

Citing articles: 4 View citing articles $\square$ 
XIX. A Study of the Ionization produced by $\beta$ and $\gamma$ Rays at High Pressures. By D. C. H. Flonance, M.A., M.Sc.*

\section{[Plate I.]}

TVHE ionization produced by $\gamma$ rays in a closed ressel is 1 due partly to the $\beta$ rays escaping from the walls, and partly to the $\beta$ rays set up from the molecules of the gas. There is no definite proof that $\gamma$ rays ionize directly. Kleeman (Proc. Roy. Soc. March 1909) has attempted to separate the wall effect from the gas effect by means of the magnetic deflexion of the $\beta$ rays from the wall. $\mathrm{He}$ has also made a determination of the velocities and penetrating power of the "emergent" and "incident" secondary $\beta$ rays produced by $\gamma$ rays, Laby and Kaye (Phil. Mag. Dec. 1908), working with pressures up to 20 atmospheres, and Wilson (Phil. Mag. Jan. 1909) with pressures up to 40 atmospheres, have investigated the effect of pressuro on the ionization produced by $\gamma$ rays in a closed vessel.

Kovarik (Abstract Phys. Rev. Feb. 1912), by increasing the pressure of the air in his ionization-chamber to about 20 atmospheres, has found the absorption coefficient of the $\beta$ rays from RaE to be $0.0149 \mathrm{~cm} .^{-1}$ air, and of the harder $\beta$ rays from $R a D$ to be $0.094 \mathrm{~cm}^{-1}$ air. The writer has found the absorption coefficient of the $\beta$ rays from $\mathrm{UrX}$ to be $0.007 \mathrm{~cm}^{-1}$ air.

In the present paper a pressure of 80 atmospheres has been attained, and the apparatus has been so arranged that the $\beta$ rays emitted from different metals could bo examined. It was thus hoped that the absorption coefficients of the "emergent" and "incident" secondary $\beta$ rays could be determined, and an estimate made of the relatiye number of $\beta$ particles from the walls and from the gas contained in the ionization vessel. On account of the impossibility of securing a definite cone of $\gamma$ rays and the incompleteness of certain experimental data, these quantities could only be approximately determined.

\section{Apparatus.}

The ionization vessel (fig. 1) was cylindrical, $7 \cdot 4 \mathrm{~cm}$. in diameter and $27.5 \mathrm{~cm}$. in height. The pressure, which could be raised to 80 atmospheres, was measured by a carefully calibrated steel tube-gauge. $A$ is a compound plate

* Communicated by Prof. E. Rutherford, F.R.S. 


\section{Mr. D. C. H. Florance : $A$ Study of the Ionization}

A balance method, sinilar to that described by Townsend (Phil. Mag. May 1903), was used in these experiments for measuring the ionization currents. This method was found to be far more accurate and certain than the ordinary rate method. A Dolazalek electrometer of sensibility about $400 \mathrm{~mm}$. divisions per volt was used. The experimental arrangement is shown in fig. 1 .

\section{Variation of Current with Voltage.}

It is important that at each given pressure the saturation current should be measured ; but it was found quite impossible to obtain complete saturation, even at low pressures, with a voltage of 2200 . In fig. 2 ( $\mathrm{Pl}$. I.) the saturation curves for the ionization due to the $\beta$ rays from Ur $\mathrm{X}$ are shown when the pressure in the ionization-chamber was raised from 5 to 70 atmospheres, and the voltage was increased from 80 to 1800 volts. Although for no pressure was complete saturation obtained, it should be noticed that all the curves are of the same character, and that there is a constant ratio between the ionization currents at any two given pressures when the voltage is constant. It would therefore appear that the true saturation current at any pressure is a definite multiple of the ionization current measured for a particular voltage. The shape of the saturation curves due to $\beta$ rays at high pressures resembles those obtained for $\alpha$ rays at ordinary pressure. There appears to be the same difficulty in obtaining complete saturation. The saturation curves obtained when $\gamma$ rays were used as the source of ionization were similar to those obtained for the $\beta$ rays of $\mathrm{Ur} X$.

An experiment was then carried out to see whether the saturation curves depended on the activity of the source. Two definite pressures, 5 and 25 atmospheres, were chosen. The saturation curves were plotted when the voltage was increased from 80 to 1800 volts. By a suitable adjusiment of the lead screens in front of the radium, the ionization current for a given voltage and pressure was doubled. It was found that the ionization current was also doubled for all voltages at both pressures.

The numbers quoted by Laby and Kaye (Phil. Mag. Dec. 1908) seem to support the above results, except that they got complete saturation at 1500 volts for a pressure of 8 atmospheres and at 3500 volts for a pressure of 15 atmospheres. The writer had not available higher voltages than 2200 , and was unable in any case to obtain evidence of complete saturation. In the experiments to be described later, 
it has been assumed that no sensible error in the relative values of the ionization currents has been introduced by using a voltage of 1520 when the plates were $1 . \mathrm{cm}$. apart.

\section{Absorption by Air of the $\beta$ rays from $\operatorname{Ur} X$.}

When $\gamma$ rays pass through any material some of the $\gamma$ rays are absorbed and $\beta$ rays are consequently produced. "Emergent" $\beta$ rays continue in the direction of the original $\gamma$ rays, and "incident" $\beta$ rays are returned in the opposite direction. In order to obtain some idea of what happens to the emergent and incident $\beta$ rays in the ionization vessel when the pressure was increased, a comparison was made with the $\beta$ rays of $\mathrm{UrX}$. This active material was chosen becanse a thin film could be easily prepared of any desired area, and the $\beta$ rays correspond approximately in penetrating power to the $\beta$ rays from Radium C.

A uniform layer of $\mathrm{UrX}$ was deposited on platinum foil abont $3 \mathrm{~cm}$. in diameter. This was covered with aluminium $0.2 \mathrm{~mm}$. thick to absorb soft $\beta$ rays. This active material was fixed to the lower plate. The upper plate was aluminium foil $(0 \cdot 00024 \mathrm{~cm}$. thick $)$, so that reflexion in the case of the upper plate was reduced to a minimum; yet there would undoubtedly be a certain amount of reflexion from the interior of the vessel, and this it was impossible to. estimate. The distance between the plates was $1 \mathrm{~cm}$. The pressure in the vessel was increased, and the ionization currents were measured for the different pressures. The results are shown graphically in fig. 3 (Pl. I.). Assuming that ionization and absorption are proportional to the pressures, the saturation current at any pressure should be given by

$$
i=\mathrm{A}\left(1-e^{-p \lambda d}\right)
$$

where $\mathrm{A}=\mathrm{a}$ constant;

$p=$ pressure in atmospheres ;

$\lambda=$ coefficient of absorption it unit pressure ;

$d=$ distance apart of the plates.

The curve, fig. 3, can be expressed approximately by an equation of this type, and the value of $\lambda$ was thus found to be $0.04 \mathrm{~cm} .^{-1}$. If we calculate the absorption coefficient of the $\beta$ rays in air from the known absorption coefficient in aluminium, assuming the density law, $\lambda=0.0064$ approximately. 'This discrepancy between the observed value and the calculated value cannot be ascribed to lack of saturation at the high pressures. 


\section{Mr. D. C. H. Florance : A Study of the Ionization}

Consider the shape of the ionization-chamber and the consequent paths of the $\beta$ rays. Each plate is $7 \mathrm{~cm}$. in diameter, the distance apart is $1 \mathrm{~cm}$., and the active material is spread over a surface $3 \mathrm{~cm}$. in diameter. If the $\beta$ rays pass out equally in all directions, not more than 20 per cent. of the total number of $\beta$ rays emitted will traverse a distance less than $1.3 \mathrm{~cm}$.; and about 30 per cent. will have a path of $3.5 \mathrm{~cm}$. in air. Hence, instead of the effective distance between the plates being $1 \mathrm{~cm}$., the average path between the plates, allowing for the obliquity of the rays, is roughly $2 \cdot 2 \mathrm{~cm}$. Allowing for this correction, the value of the absorption coefficient should be $\cdot 014$, instead of the calculated value $\cdot 0064$. The experimental value is, however, $\cdot 04 \mathrm{~cm}^{-1}$.

No account has been taken of scattering and multiple reflexion, and it is obvious that these factors are of great importance in the present case. Since the upper plate is Al foil, the $\beta$ rays reflected from the walls and top of the vessel will have a certain ionizing effect on the gas between the plates. It is difficult to evaluate this effect. The number of $\beta$ rays striking the walls decrease as the pressure in the vessel increases; but the ionization due to these reflected $\beta$ rays will rise to a maximum and then diminish, The $\beta$ rays turned back by the air above the Al foil will first increase to a maximum with pressure, and then decrease on account of the absorption of the $\beta$ rays between the plates. The ionization due to these $\beta$ rays will increase to a maximum and finally decrease as the pressure is raised. There will also be a small effect due to the $\beta$ rays finally reflected back from the plate carrying the active material. A certain number of the primary $\beta$ rays that penetrated the bottom plate will be scattered back into the gas. Wilson (Proc. Roy. Soc. March 1912) hus recently shown that the absorption coefficient in aluminium of reflected $\beta$ particles may have average values ranging from 14 to 40 .

Hence, taking into consideration scattering and multiple reflexion and the consequent variation of ionization due to increase of pressure, the discrepancy between the experimental and the calculated value of the absorption coefficient can be explained. The curve (fig. 3) can be resolved into its various components in order to allow for the separate $\beta$-ray effects, but the data at present available are too incomplete to give definite values for all the factors involved.

An experiment was made to show definitely that the high value obtained for the absorption coefticient was due mainly 
to the obliquity and the scattering of the $\beta$ rays. The $\mathrm{Ur} X$ was placed at the bottom of the ionization-chamber and covered with $0.1 \mathrm{~mm}$. of aluminium. At a distance of $4.3 \mathrm{~cm}$. above the active material was a lead plate, $2 \cdot 3 \mathrm{~mm}$. thick, with a circular opening $3 \mathrm{~cm}$. in diameter. Over this opening was fastened a sheet of aluminium, $0.1 \mathrm{~mm}$. thick, to absorb soft scattered radiation. The upper plate was Al foil, and the distance apart was $1.2 \mathrm{~cm}$. The lead plate and aluminium covering were charged to 1520 volts.

As the pressure in the vessel was raised the ionization between the plates increased until it reached a maximum and then decreased. The distance between the active material and the bottom plate being $4.3 \mathrm{~cm}$., and the circular opening in the lead and the surface of the active material being both $3 \mathrm{~cm}$. in diameter, the direct distance that the $\beta$ rays had to traverse was between $4 \cdot 3$ and $5 \mathrm{~cm}$. in the lower chamber, and between 1.2 and $1.3 \mathrm{~cm}$. between the plates.

The corresponding problem for $\alpha$ rays has been given by Rutherford in 'Radio-Activity.'

The saturation current is proportional to

$$
\frac{\mathrm{I}_{0}}{\lambda}\left(e^{-p \lambda d_{1}}-e_{2}^{p \lambda d}\right) \text {. }
$$

This is a maximum when

$$
\log _{e} \frac{d_{1}}{d_{2}}=-p \lambda\left(d_{2}-d_{1}\right)
$$

From this expression

$$
\lambda=\cdot 0076 .
$$

Allowing for the obliquity of the rays, $\lambda$ would be about .007 . It should be noticed in fig. 4 that at 80 atmospheres, when the $\beta$ rays have passed through a distance equivalent to $350 \mathrm{~cm}$. at normal pressure, there is a considerable ionization effect between the two plates. Substituting $\lambda=\cdot 0076$ in the expression for the saturation current and plotting the curve, there is a fair agreement between the theoretical and experimental curves.

The experiments with $\mathrm{UrX}$ were in progress for over a fortnight, so that the decay of $U r X$ was also examined. For any definite pressure the period of Ur X was found to be about twenty-two day's.

When the Ur X was covered with $\cdot 4 \mathrm{~mm}$. of Al the ionization-pressure curve was similar to that obtained when the material was covered with $2 \mathrm{~mm}$. of Al. The absorption

Phil. Mag. S. 6. Vol. 25. No. 145. Jan. 1913. N 
178 Mr. D. C. H. Florance : A Study of the Ionization

coefficient of the $\beta$ rays in aluminium (between $\cdot 2$ and $-4 \mathrm{~mm}$.) for any pressure was approximately $19 \mathrm{~cm}^{-1}$, whereas in the usual experimental arrangement the value is 14 .

\section{The Effect of the Final Plate on the Amount of Emergent $\beta$ Radiation.}

The $\gamma$ rays passing into the ionization-chamber were atways approximately of the same intensity. The ionization between the plates at atmospheric pressure should be mainly dependent on the nature of the plates. Since at atmospheric pressure the ionization currents were extremely small, another experiment was performed to see how the quantity of emergent $\beta$ rays depended on the material of the plates.

Fig. 5 illustrates the experimental arrangement. The ionization-chamber (kindly lent by Mr. Moseley) was constructed of Al foil supported on knitting-needles. It was

Fig. 5.
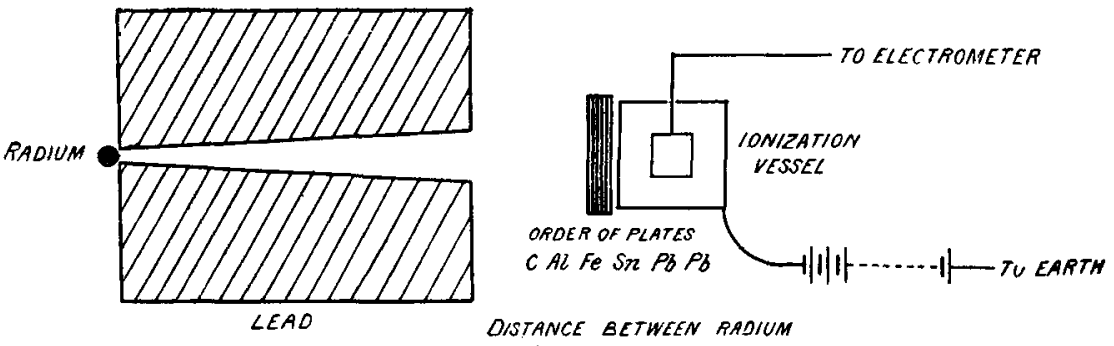

ONTANCE BETWEEN RAOIUM

AND IONIZATION VESSLL 60 CMS.

about $7 \cdot 3 \mathrm{~cm}$. cube. This ionization-chamber rested on thin glass tubes. Against the side of the ionization-chamber nearest the radium was arranged the following series of plates :-

\begin{tabular}{|c|c|c|c|c|c|c|}
\hline Plate ... & Carbon. & Aluminium. & Iron. & Tin. & Lead. & Lead. \\
\hline $\begin{array}{c}\text { Thickness } \\
\text { in mm. }\end{array}$ & 4.9 & 3.4 & $2 \cdot 1$ & $2 \cdot 0$ & $4 \cdot 3$ & 9.2 \\
\hline
\end{tabular}

Between the plate of lead $9 \cdot 2 \mathrm{~mm}$. thick and the vessel there was just sufficient space to admit one of the other plates, the $\beta$ rays of which were to be examined. The $\gamma$ rays always passed through the same quantity of material; hence, in each case the effect observed was chiefly due to 
the $\beta$ rays emerging from the plate next to the ionizationchamber. The plate of lead $\mathbf{9 \cdot 2} \mathrm{mm}$. thick was placed near the vessel to reduce to a minimum the effect of the scattering in the substances of smaller atomic weights. The curve (fig. 6, Pl. I.) is similar to that obtained by Bragg (Phil. Mag. Dec. 1908).

\section{The Ionization due to the $\gamma$ Rays of Radium.}

The experimental arrangement is shown in fig. 1. The source of $\gamma$ rays was 30 mgrs. of radium bromide. It is impossible to secure a perfect cone of $\boldsymbol{\gamma}$ rays on account of the scattering they undergo whenever they traverse matter. Precautions were, however, taken to obtain an approximate cone of rays. A conical opening was cut in a large block of lead, and the radium was placed in a definite position at the apex. Four lead slides, each $3 \mathrm{~mm}$. thick, were placed in the positions marked in order to cut out the softer $\boldsymbol{\gamma}$ rays. Practically all the $\gamma$ rays entering the ionization-vessel proceed from this conical opening. The space between the plates is traversed by the main cone of $\gamma$ rays together with those $\gamma$ rays scattered from the original cone by the plates. Besides the $\beta$ rays produced by the $\gamma$ rays from the two plates, there are also $\beta$ rays set up in the walls of the vessel due to the scattered $\gamma$ rays.

Experiments were carried out-

(1) Keeping the bottom plate the same and varying the upper plate- $\mathrm{Pb}, \mathrm{Al}$, or Al-foil ;

(2) Keeping the upper plate the same and reversing the bottom plate $\mathrm{Al}$ or $\mathrm{Pb}$;

(3) Varying the distance between the plates in each of the two previous arrangements.

Fig. 7 (Pl. I.) shows the results obtained when the plates were kept at a constant distance of $1 \mathrm{~cm}$. apart. In curve $A$ the lower plate was aluminium and the upper plate lead, and in curve $C$ the lower plate was lead. When the $\gamma$ rays pass through $1.5 \mathrm{~cm}$. of lead there are more emergent $\beta$ rays from aluminium than from lead. Hence, if the curve $C$ is subtracted from the curve $A$, or $D$ from $B$, then the difference should give the approximate effect of the additional $\beta$ rays from the aluminium plate. The difference between the curves gives an indication of what happens to a certain proportion of the corpuscular emergent radiation when the pressure is varied. Since more $\beta$ rays are scattered from a $\mathrm{N} 2$ 
plate of lead than from a plate of aluminium, the curve $(A-C)$ is greater than $(B-D)$. From the curve $(A-C)$ (fig. 8) the apparent coefficient of absorption of the $\beta$ rays is observed to be approximately $0.046 \mathrm{~cm}^{-1}$; and it has already been shown that the absorption coefficient of the $\beta$ rays from $\mathrm{UrX}$ determined under similar conditions was $0.04 \mathrm{~cm} .^{-1}$.

There are more "incident" $\beta$ rays ejected from lead than from aluminium; hence the difference between the curves $A$ and $B$, or $C$ and $D$, will give a general indication of the behaviour of a certain proportion of the incident $\beta$ rays. The coefficient of absorption of these incident $\beta$ rays $=$ $0.056 \mathrm{~cm} .^{-1}$ approximately. The emergent $\beta$ rays appear to be more penetrating than the incident $\beta$ rays; and this is to be anticipated just as in the case of scattered $\beta$ rays, which are more absorbable the greater the angle through which they have been deflected.

Beatty (Phil. Mag. August 1910) has found that the incident corpuscular radiation produced by a homogeneous beam of $\mathrm{X}$ rays was similar in penetrating power to the emergent corpuscular radiation.

When the upper plate was aluminium foil and the lower plate changed from lead to alumininm, the curves were similar to those in which aluminium was the upper plate. 'The absorption coefficient for the emergent $\beta$ rays in this case was $0.04 \mathrm{~cm}^{-1}$.

In all these experiments the $\beta$ radiation appears to be independent of the plates except for multiple reflexion; and the emergent radiation rather more penetrating than the incident.

The curves of fig. 7 are all concave to the pressure axis. Bragg predicted that with a light substance for the upper plate the curve should be convex to the pressure axis. An attempt was made to obtain this condition by making the upper plate of Al foil and having a column of air $24 \mathrm{~cm}$. high above the plate. In order to reduce still further the scattered $\beta$ rays, the top of the vessel was lined with carbon and the side of the vessel lined with ebonite coated with Al foil. Of course this has not reduced the problem to the simple one that Bragg assumes-namely, no radiation from the sides of the vessel and none from the upper plate. The result thus obtained is similar to that in which an aluminium plate was used. At lower pressures the ionization current is slightly greater for aluminium foil, and at the high pressures less than the current given when the upper plate is aluminium. Before discussing the curves further let us briefly consider the theory of the ionization by $\gamma$ rays. 
The theory for a large shallow ionization-chamber has been worked out by Bragg (Phil. Mag. Sept. 1910). The lower plate was supposed to be composed of some dense material, and the upper plate of some material that would reflect very few $\beta$ rays. Hence, using Bragg's notation, we have :-

I. The wall effect, i.e., the ionization produced in the chamber by the emergent $\beta$ rays,

$$
=k I\left(1-e^{-\lambda^{\prime} D}\right) / \lambda
$$

where

$D=$ depth of chamber $\times$ density,

$\lambda=$ weight absorption coefficient of $\beta$ rays in the plate,

\begin{tabular}{|c|c|c|c|}
\hline$\lambda^{\prime}=$ & " & " & $" \quad$ in the gas, \\
\hline$k^{\prime}=$ & , & $"$ & $\gamma$ rays in the gas, \\
\hline$k=$ & , & " & in the plate. \\
\hline
\end{tabular}

II. The gas effect

$$
=k^{\prime} \mathrm{DI}-k^{\prime} \mathrm{I}\left(1-e^{-\lambda^{\prime} \mathrm{D}}\right) / \lambda^{\prime} .
$$

The total ionization in the chamber

$$
=\mathrm{I}\left\{\mathrm{D} k^{\prime}+\left(\frac{k}{\lambda}-\frac{k^{\prime}}{\lambda^{\prime}}\right)\left(1-e^{-\lambda^{\prime} \mathrm{D}}\right)\right\} \text {. }
$$

In the present experiments it was found impossible to reproduce such simple conditions. A complete expression representing the action of the $\gamma$ rays is very complex, and the value of the constants too uncertain to make such an expression of any value in the present case. It is necessary to take into consideration the effect of the $\beta$ rays set up in the sides of the vessel due to the scattered $\gamma$ rays and the effect of multiple reflexion on the resultant ionization. Again, the indirect ionization produced by the $\gamma$ rays in the gas will depend, to a certain extent, on the lower and upper plates. A lead plate will always return more $\beta$ rays into the ionization-chamber than will a plate of aluminium. There is in fig. 7 a slight difference in the final slope of the curves, but the pressure has not been raised sufficiently high to determine how far this is dependent on the plates.

From figs. 3 and 8 it is seen that the $\beta$ ray curve due to $\mathrm{Ur} \mathrm{X}$ is similar to that obtained by plotting the differences in the ionization currents when the bottom plate is changed 


\section{Mr. D. C. H. Florance: A Study of the Ionization}

from $\mathrm{Al}$ to $\mathrm{Pb}$. Hence we conclude that the $\beta$ rays from the walls of the vessel are similar to the $\beta$ rays from Ur X. With y rays we have not only this "wall effect," but we have also the "gas effect"; and this shows itself in the final slope of the curve, which at 80 atmospheres is still increasing, and at a rate apparently dependent on the material of the plates.

Taking the simplest case, that of two plates of aluminium $1 \mathrm{~cm}$. apart, curve B (fig. 7), between 0 and 20 atmospheres, is similar to that obtained by Laby and Kaye (Phil. Mag. Dec. 1908) when the sides of their ionization-chamber were aluminium and at a distance $1.1 \mathrm{~cm}$. apart. Assume that the average coefficient of absorption of the $\beta$ rays proceeding from the walls is equal to 0.04 , which was the experimental value found for the $\beta$ rays of $U r X$. The ionization-pressure curve for these $\beta$ rays reaches a maximum at about 80 atmospheres. For $\gamma$ rays, when the plates are $1 \mathrm{~cm}$. apart, the ionization due to the $\beta$ rays set up in the gas should be small at 5 atmospheres. If we assume, then, that most of the ionization in the gas at this pressure is due to the $\beta$ rays from the walls, the ionization at the other pressures can be represented by the curve $x$ of fig. 9 . The difference between the curves $\mathrm{B}$ and $x$ is the curve $y$, which consequently represents the value of the ionization due to the $\gamma$ rays in the gas at the different pressures. It seems improbable that the effect due to the $\gamma$ rays in the gas is smaller than that represented by the curve $y$.

The "gas effect" is given by the expression

$$
k^{\prime} \mathrm{DI}-k^{\prime}\left(1-e^{-\lambda^{\prime} \mathrm{D}}\right) / \lambda^{\prime},
$$

where $k^{\prime}$ and $\lambda^{\prime}$ are the mass absorption coefficients for $\gamma$ and $\beta$ rays respectively. A correction has to be made for the ionization due to the $\beta$ rays that are reflected from the $\mathrm{Al}$ plates. It is difficult to decide what values ought in this case to be given to the absorption coefficients of the $\beta$ rays before and after reflexion, and also the value to be assigned to the constant of reflexion. By an arbitrary choice of these values the curve $y$ can be obtained.

To determine the maximum limit of the $\gamma$ ray effect, a tangent is drawn to the curve $\beta$ at 80 atmospheres and the line $\mathrm{O} z$ is drawn parallel to it. The straight line $\mathrm{O} z$ would represent the ionization due to the $\gamma$ rays in the gas if the bottom plate had been the same density as air. Since the bottom plate has cut out the $\beta$ rays that would have come 
from the corresponding plate of air, the true gas effect must be represented by a curve the ordinates of which are considerably less than those of $\mathrm{O} z$. When the aluminium plates are $1 \mathrm{~cm}$. apart, it is probable that at 80 atmospheres not more than 25 per cent. of the total ionization is due to $\gamma$ rays producing $\beta$ rays in the gas, and that at atmospheric pressure the effect is negligible.

When the plates are $2 \mathrm{~cm}$. apurt (fig. 10), the apparent absorption coefficient of the emergent $\beta$ rays is equal to $0.025 \mathrm{~cm}^{-1}$ approximately, and under similar conditions the absorption coefficient of the $\beta$ rays from $\operatorname{UrX}=0.025 \mathrm{~cm}^{-1}$. Hence it would appear that the $\beta$ rays produced by $\gamma$ rays are similar in penetrating power to the $\beta$ rays of $U \mathrm{r} X$. The high values obtained for the absorption coefficients in these experiments are due to the obliquity of the paths of the rays, and to the multiple reflexion taking place in the chamber.

\section{Summary.}

1. The apparent coefficient of absorption of the $\beta$ rays from Ur X was found to be $0.04 \mathrm{~cm}^{-1}$ air. By a special arrangement this value could be reduced to 0.007 approximately.

2. The "emergent" $\beta$ rays produced by $\gamma$ rays from RaC havean absorption coefficient $0.046 \mathrm{~cm}^{-1}$ air, and the incident $\beta$ rays $0 \cdot 056 \mathrm{~cm}^{-1}$ air. The emergent $\beta$ rays are similar in penetrating power to the $\beta$ rays of Ur X.

3 . The absorption coefficient of the $\beta$ rays of $U r X$, and of the emergent $\beta$ rays when the plates are $2 \mathrm{~cm}$. apart, is $0.025 \mathrm{~cm}^{-1}$ approximately.

4. The $\beta$ rays appear to be independent of the material of the plates, as other investigators have also shown.

5 . When the plates are $1 \mathrm{~cm}$. apart the ionization due to $\gamma$ rays in the gas is negligible at atmospheric pressure, and is approximately 25 per cent. of the total ionization at 80 atmospheres.

I wish to thank Prof. Rutherford for suggesting this research, and for his advice during the course of these experiments. 

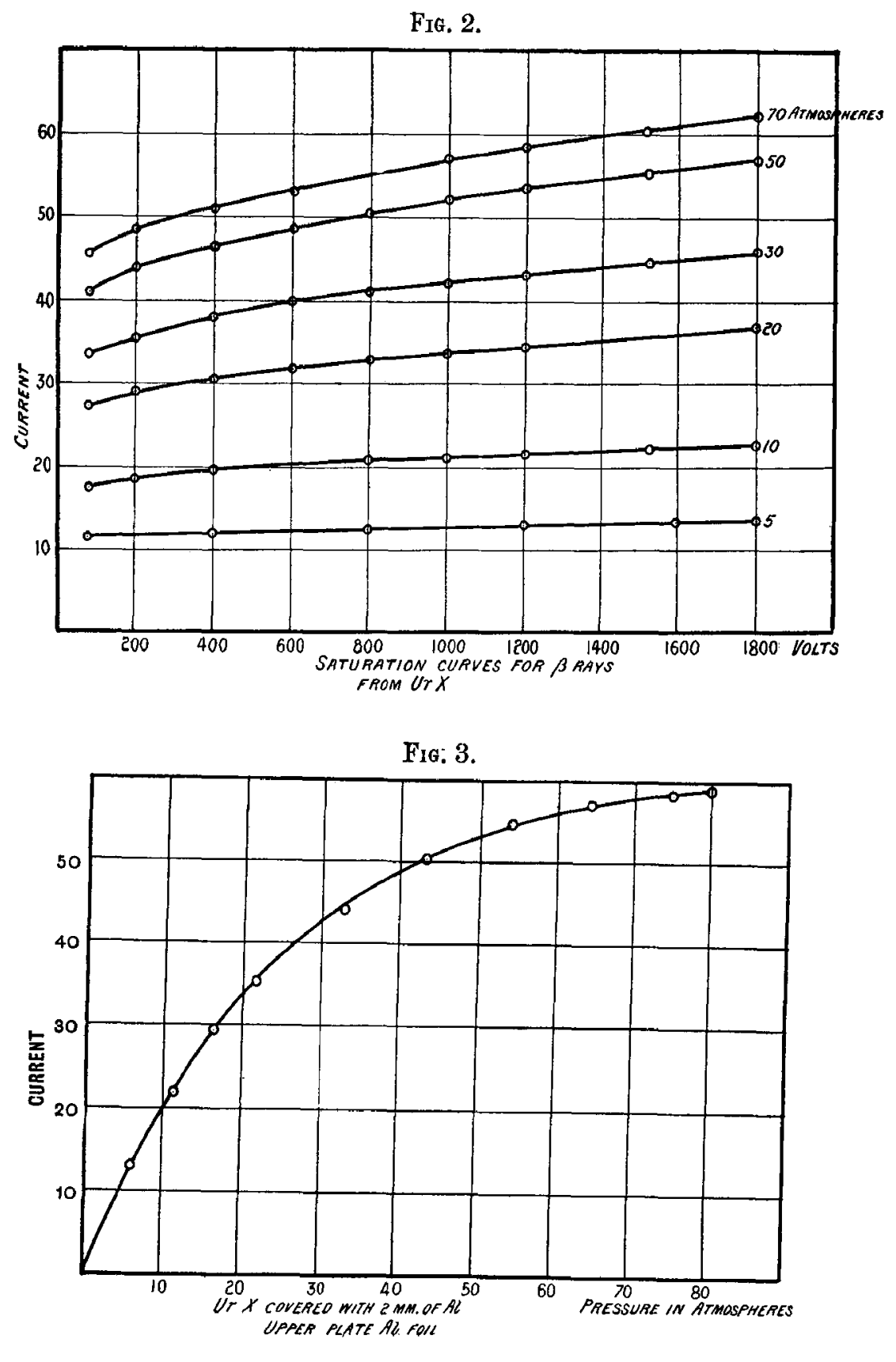
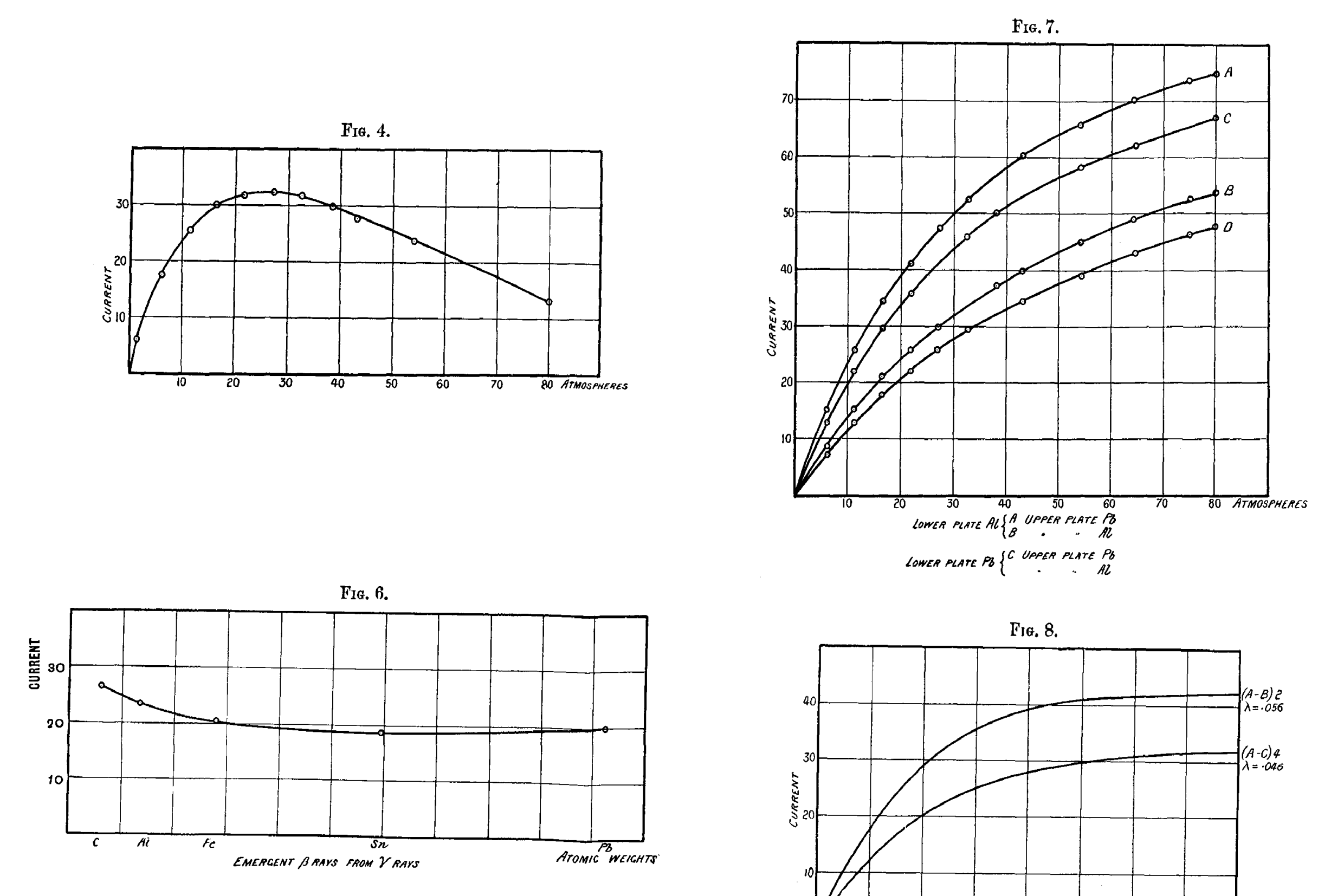

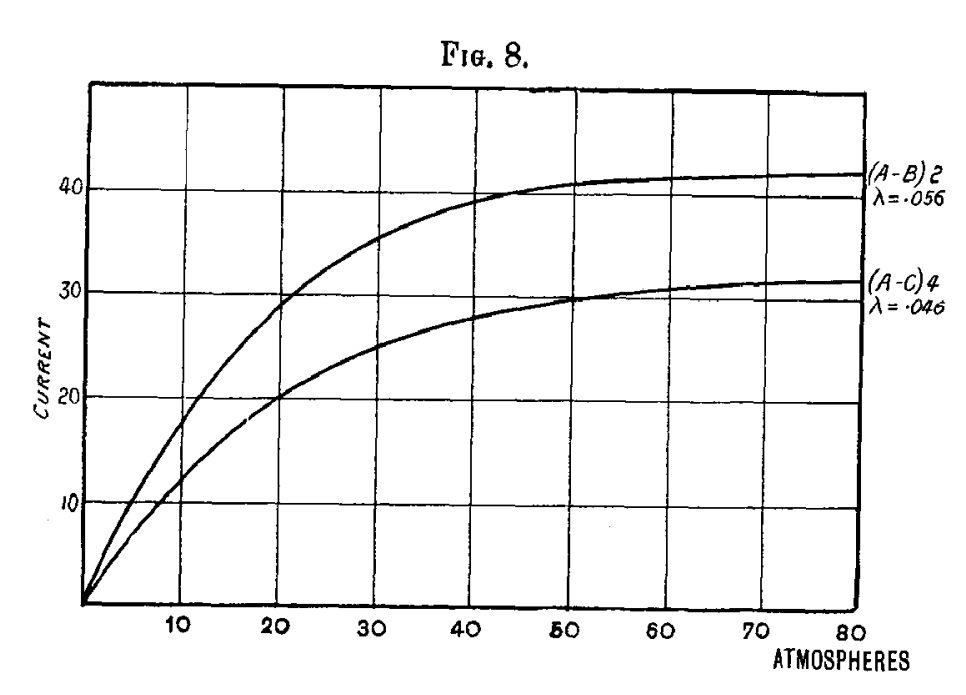

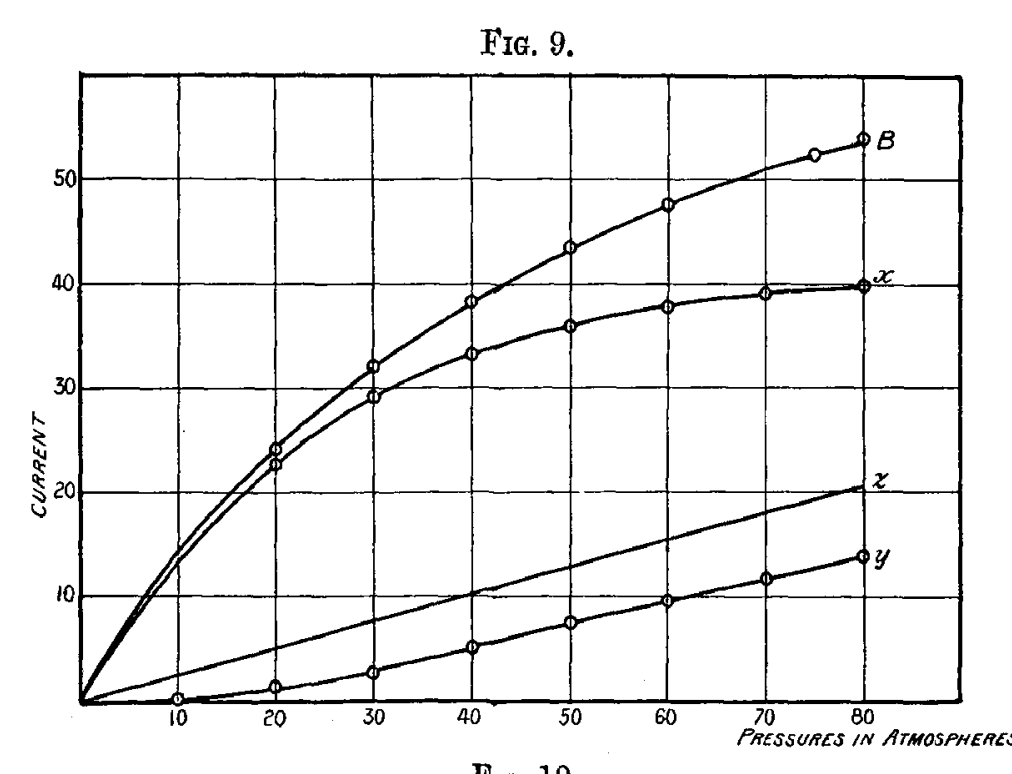

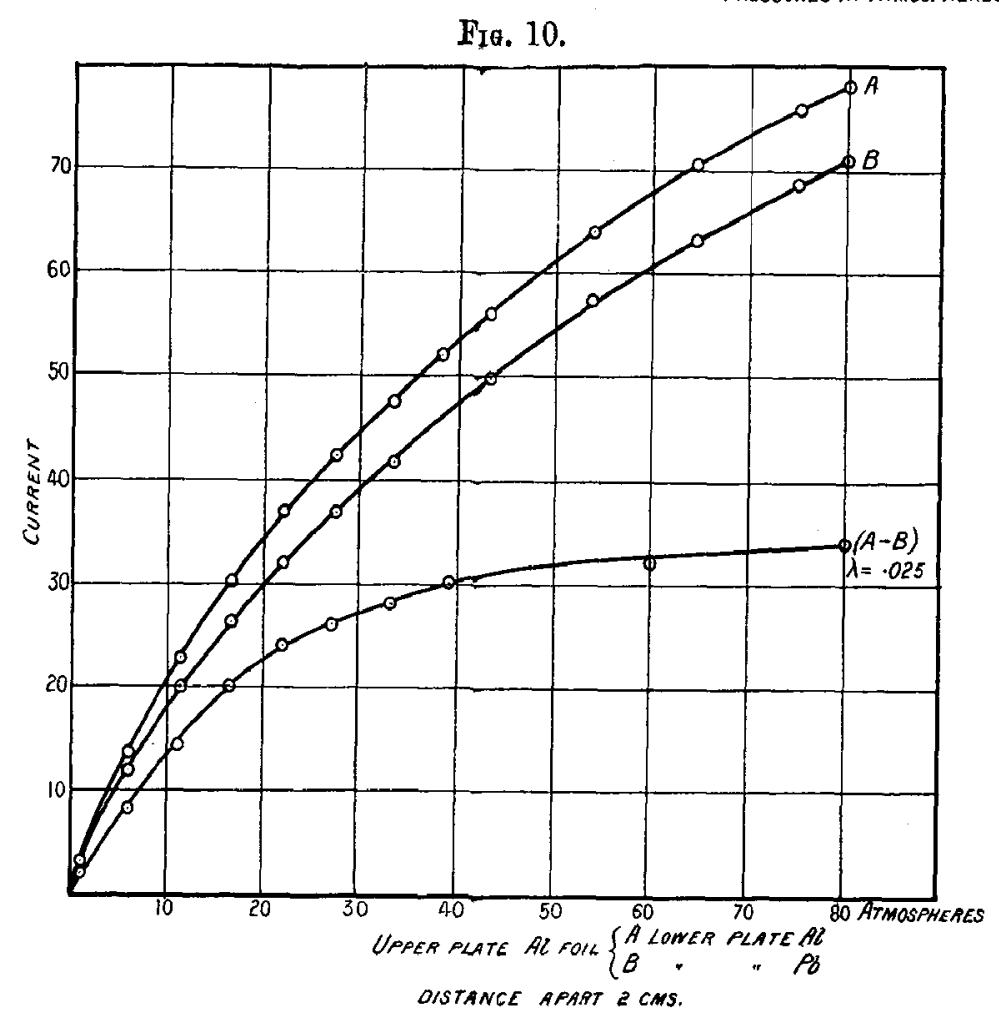

